\title{
Probing the Anomalous Flux of Very-high-energy Gamma rays from the Sun with HAWC
}

\author{
Mehr Un Nisa* \\ For the HAWC Collaboration ${ }^{\dagger}$ \\ https://www.hawc-observatory.org/collaboration/icrc2019.php \\ Michigan State University \\ E-mail: nisamehr@msu.edu \\ Segev BenZvi \\ University of Rochester \\ E-mail: sybenzvi@pas.rochester.edu
}

\begin{abstract}
The Sun has been observed by Fermi-LAT as a surprisingly bright source of gamma rays up to hundreds of $\mathrm{GeV}$. The gamma rays can be attributed to interactions of cosmic rays in the solar atmosphere. Certain models of solar dark matter capture and annihilation also predict steady gamma-ray emission from the Sun. Interestingly, at GeV energies the flux observed by FermiLAT is much brighter ( $>$ factor of 6 ) and spectrally harder than predicted from cosmic-ray interactions. The flux also shows a dependence on solar activity, with the highest energy photons occurring during the solar minimum and exhibiting no spectral cutoff up to at least $200 \mathrm{GeV}$. Observations of the Sun above $1 \mathrm{TeV}$ may shed light on the mechanisms which produce the unexplained gamma rays. Non-observations of gamma-rays $>1 \mathrm{TeV}$ can be used to constrain cosmic-ray interactions, and provide limits on dark matter annihilation inside the Sun. The High Altitude Water Cherenkov Observatory (HAWC) is currently the only detector capable of monitoring the Sun at energies above $1 \mathrm{TeV}$. HAWC observations placed strong constraints on steady $\mathrm{TeV}$ emission from the Sun during the active phase of solar cycle 24, demonstrating sensitivity to an energy flux of $\sim 10^{-12} \mathrm{TeV} \mathrm{cm}{ }^{-2} \mathrm{~s}^{-1}$. The Sun entered a new solar minimum beginning 2018. We present new results from 1 year of data collected by HAWC during the ongoing solar minimum and discuss the implications for models of gamma-ray emission from the Sun.
\end{abstract}

36th International Cosmic Ray Conference -ICRC2019-

July 24th - August 1st, 2019

Madison, WI, U.S.A.

\footnotetext{
* Speaker.

${ }^{\dagger}$ for collaboration list, see PoS(ICRC2019) 1177.
} 


\section{Introduction}

Cosmic rays approaching the Sun's atmosphere can be redirected by the Sun's magnetic field, resulting in gamma-ray production from hadronic cascades in the solar disk. The Fermi-LAT has been observing the Sun since 2008 [1], and has robustly detected a flux of very-high-energy gamma-ray emission extending above $100 \mathrm{GeV}$ in energy. The measured gamma-ray excess shows an anti-correlation with the solar cycle, with a hard $E^{-2.2}$ spectrum and a flux that is about 6 times higher than theoretical predictions $[2,3,4]$. In order to elucidate the underlying physics of these gamma rays, it is important to test how far the spectrum extends in the $\mathrm{GeV}-\mathrm{TeV}$ range.

The HAWC observatory started collecting data from the Sun in 2015, shortly after the solar maximum of solar cycle 24. The constraints set by HAWC on the gamma-ray flux using the first three years of data demonstrate interesting prospects for the solar minimum. If the bright flux measured by the Fermi-LAT during the last solar minimum continues into the next solar cycle with the same spectral hardness, it would be well within the sensitivity of HAWC for a first TeV detection. The Sun continues to become less active and is likely to approach a solar minimum in 2019-2020. In this proceeding, we use data collected in 2018 — as the Sun progresses towards the new minimum - to perform a search for gamma rays from the Sun. We largely follow the analysis method used in Ref. [5] and show the changing characteristics of the data from the solar disk with time.

\section{A first Solar Minimum Analysis with HAWC}

\subsection{The HAWC Observatory}

The HAWC gamma-ray experiment is an array of 300 water tanks, each instrumented with four photomultiplier tubes (PMTs) to detect extensive air showers produced by gamma rays and cosmic rays impinging on the earth's atmosphere. The array covers an area of 22,000 $\mathrm{m}^{2}$ and is located at an altitude of $4100 \mathrm{~m}$ above the sea-level in the state of Puebla, Mexico. The fraction of the array triggered during an air-shower is correlated with the energy of the shower, and the different times at which the PMTs detect a signal are used to reconstruct the direction of an air shower. More details on the detector and the data processing can be found in Ref. [6,7]. The data is divided into nine energy-proxy bins based on the fraction of the total number of PMTs that record a hit during an air shower. The HAWC observatory is sensitive to gamma-ray and cosmic-ray showers above $1 \mathrm{TeV}$ and is capable of reconstructing the direction of a shower to within $1.5^{\circ}$ in the lowest energy bins.

\subsection{The Sun Shadow in the Solar Minimum}

The Sun shadow refers to the deficit of cosmic rays observed from the direction of the Sun due to the solar disk blocking a fraction of the incoming cosmic rays from reaching the Earth. The deficit is measured with respect to the isotropic background of cosmic rays. Figure 1 shows a $10^{\circ}$ by $10^{\circ}$ region centered around the Sun at a median bin energy of $0.88 \mathrm{TeV}$. A $\sim 4 \%$ deficit of cosmic rays (relative intensity of $4 \times 10^{-3}$ ) can be seen at the position of the Sun. As demonstrated in Ref. [5], the presence of the cosmic-ray shadow in the data makes the detection of a potential gamma-ray excess more complicated. 


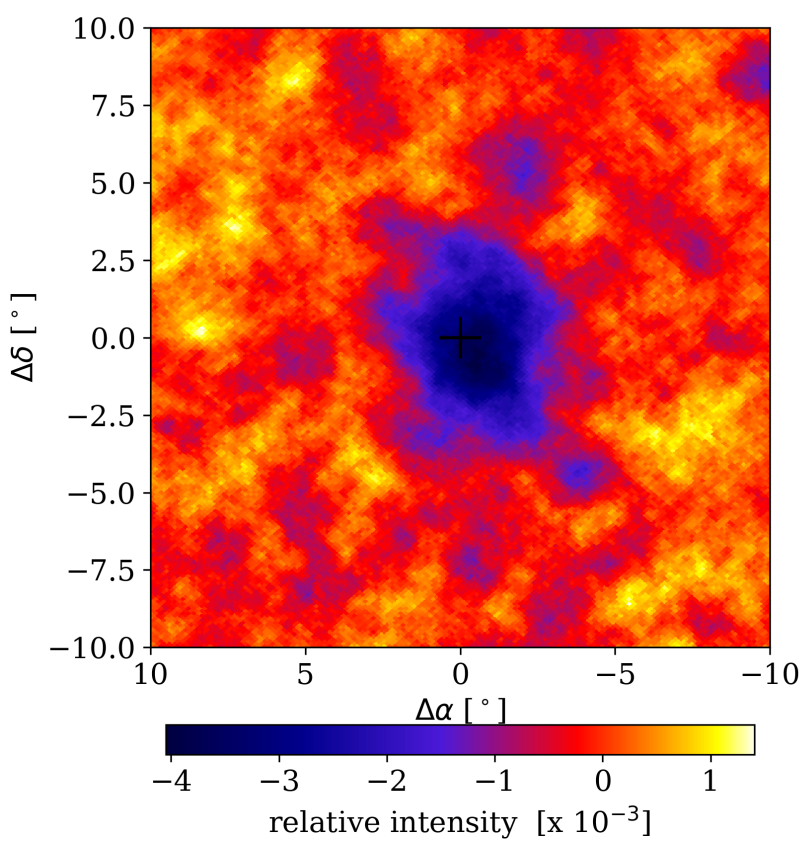

Figure 1: Sun shadow at a median bin energy of $0.88 \mathrm{TeV}$ in one year of data (2018).

In addition, the intensity of the Sun shadow also varies with the solar cycle [8]. When the Sun is active, the solar winds and the interplanetary magnetic field are able to deflect away some of the cosmic rays that penetrate the heliosphere, leading to smaller flux of cosmic rays reaching close to the Sun. To compare the intensity of the shadow in this work with previous data taken away from the solar minimum (2015-2018), we fit a to dimensional gaussian function two the shape of the Sun shadow. The best-fit amplitude of the gaussian corresponds to the maximum relative deficit at the position of the Sun (or the intensity of the shadow). Figure 2 shows the amplitude of the Sun shadow as a function of energy-proxy bin number for this work (solar minimum) and off-minimum data. It can be seen that the shadow closer to the solar minimum has a more negative amplitude for all energies, corresponding to a higher flux of cosmic rays being blocked. A detailed study of the time-variation of the Sun shadow will be the subject of future work with more data collected during the solar minimum. 


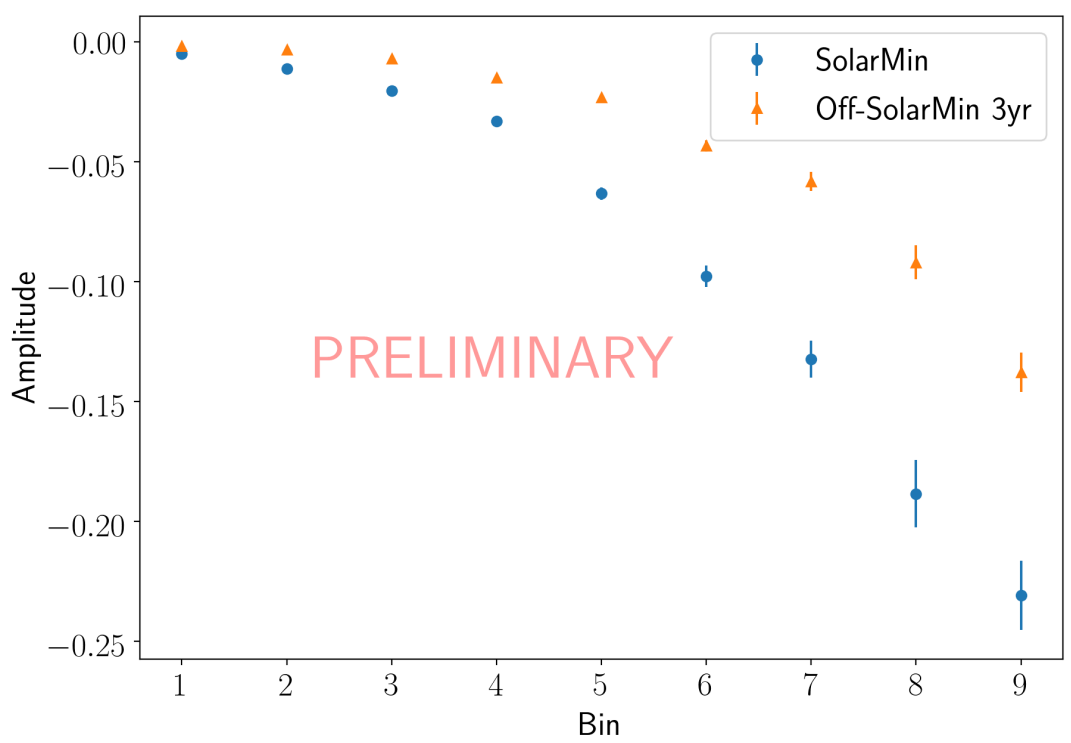

Figure 2: The amplitude of the Sun shadow quantifying the maximum relative deficit observed for each energy-proxy bin. More negative amplitudes during the solar minimum compared to the off-minimum data imply a more intense shadow.

\subsection{Estimating Gamma-ray Excess from the Sun}

In order to search for gamma rays from the solar disk, we apply gamma-hadron separation cuts to the data that selectively remove cosmic rays from the data, while keeping most of the gamma rays. At $1 \mathrm{TeV}$, the gamma-hadron cuts keep gamma rays with an efficiency of $75 \%$ and cosmic rays with an efficiency of $15 \%$. Above $10 \mathrm{TeV}$, the cosmic-ray suppression efficiency improves to $0.2 \%$. As discussed in Ref. [5], the gamma-hadron cuts reduce the significance of the shadow, which is the major limiting factor in this analysis. 


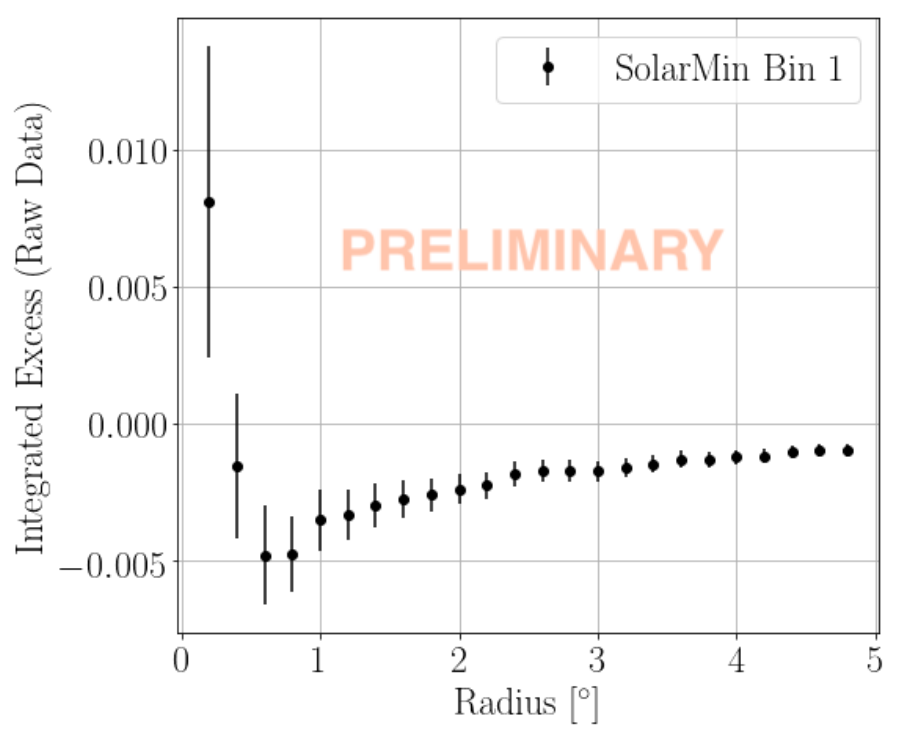

Figure 3: Net integrated fractional excess as a function of radial distance from the Sun.

Figure 3 shows the fractional excess in disks of increasing radii around the Sun in steps of $0.15^{\circ}$ for the first energy-proxy bin. A "negative excess" after the second data-point, in a disks of $\sim 0.5^{\circ}$ and greater depicts the Sun shadow. In order to account for the presence of the cosmicray deficit in the data, we need to subtract a model of the Sun shadow from the data. We use the gaussian fit to the off-minimum shadow as a model of the shadow. The off-minimum model is based on 3 years of data and its amplitude is smaller than the solar minimum shadow, making it a conservative choice. Figure 4 shows the excess in radial bins after subtracting a model of the shadow from the data in figure 3. A maximum excess of $\sim 15 \%$ is observed within $0.2^{\circ}$ of the Sun.

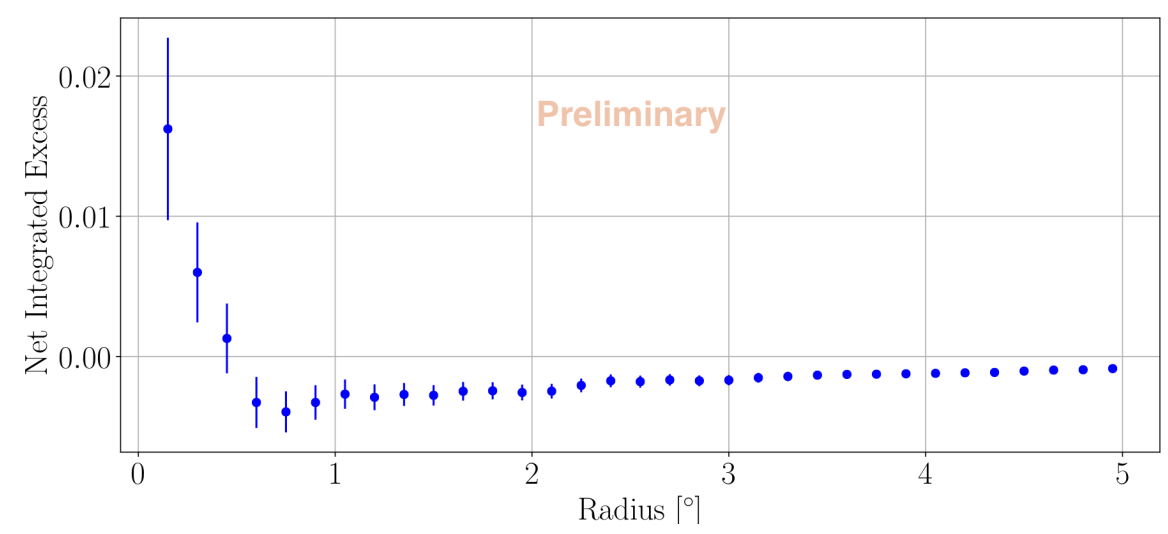

Figure 4: Net integrated fractional excess as a function of radial distance from the Sun after subtracting a model of the Sun shadow (see text for details).

Following the method in Ref. [5], we also compute the net number of gamma-ray events in the region around the Sun covered by the Sun shadow. Figure 5 shows the number of counts in the 
data with and without gamma-hadron separation, and the number of gamma-ray events divided by the total uncertainty in the full region of interest. Also, shown are the expected counts from two models of gamma-ray emission from the Sun. We also repeat the analysis on 72 off-Sun regions to obtain a sample of background-only measurements shown in the blue shaded band in figure 5 . Finding no significant excess in the on-Sun region relative to the off-Sun regions, we set upper limits on the flux from the solar disk according to the method described in Ref. [5].
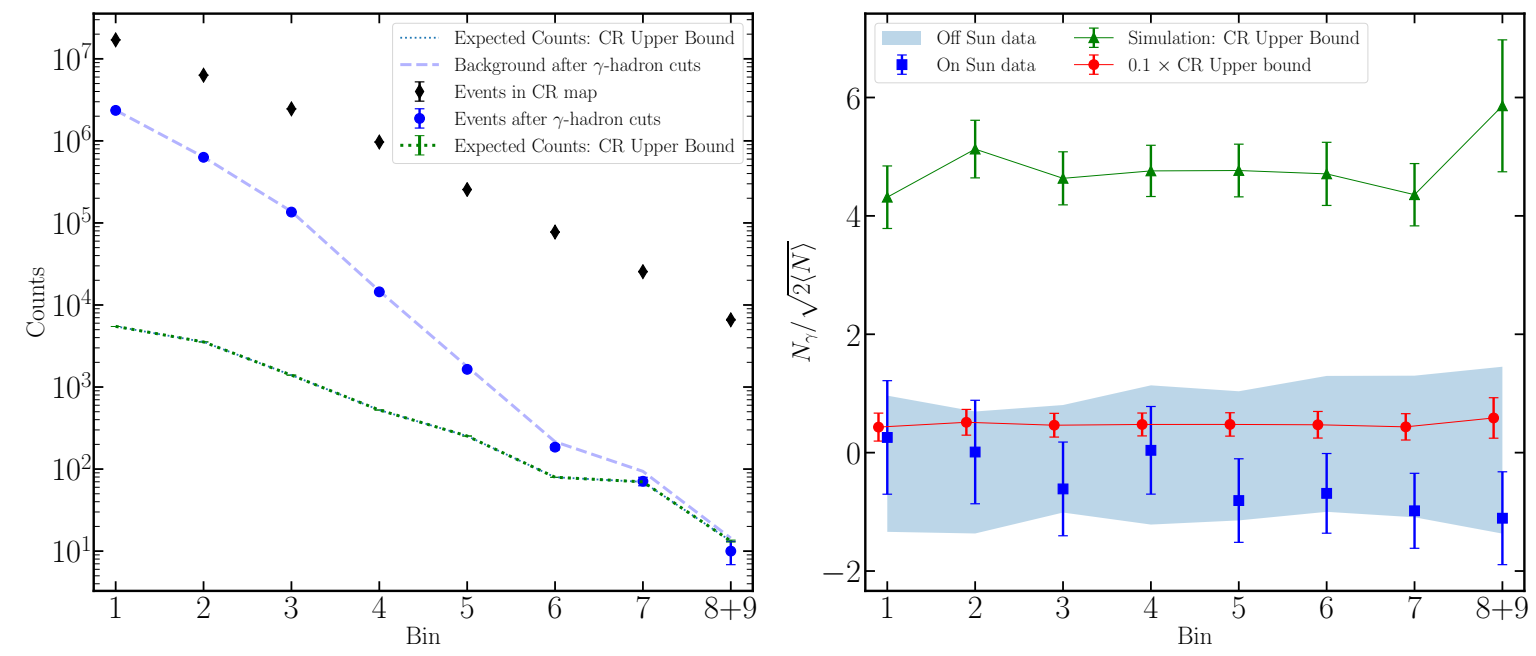

Figure 5: Left:Total number of events in the region of interest before and after applying gamma-hadron cuts to the data. Right:Net gamma-ray excess relative to an estimate of the uncertainty. The shaded blue band shows the distribution from the off-Sun regions. Also shown is the expected excess from the cosmic-ray upper bound model and $10 \%$ of the same model [5].

\section{Results}

\subsection{Initial Constraints from One year of Data}

Figure 6 shows the constraints on the energy flux from HAWC data collected during 2018. The results place upper limits on the flux at $\sim 8 \times 10^{-12} \mathrm{TeV} \mathrm{cm}^{-2} \mathrm{~s}^{-1}$ at $1 \mathrm{TeV}$. The constraints are a factor of $\sim 4$ worse than the off-minimum (2015-2017) analysis presented in Ref. [5]. Due to the small dataset the constraints do not definitively exclude a simple extrapolation of the Fermi-LAT flux measured during the last solar minimum. 


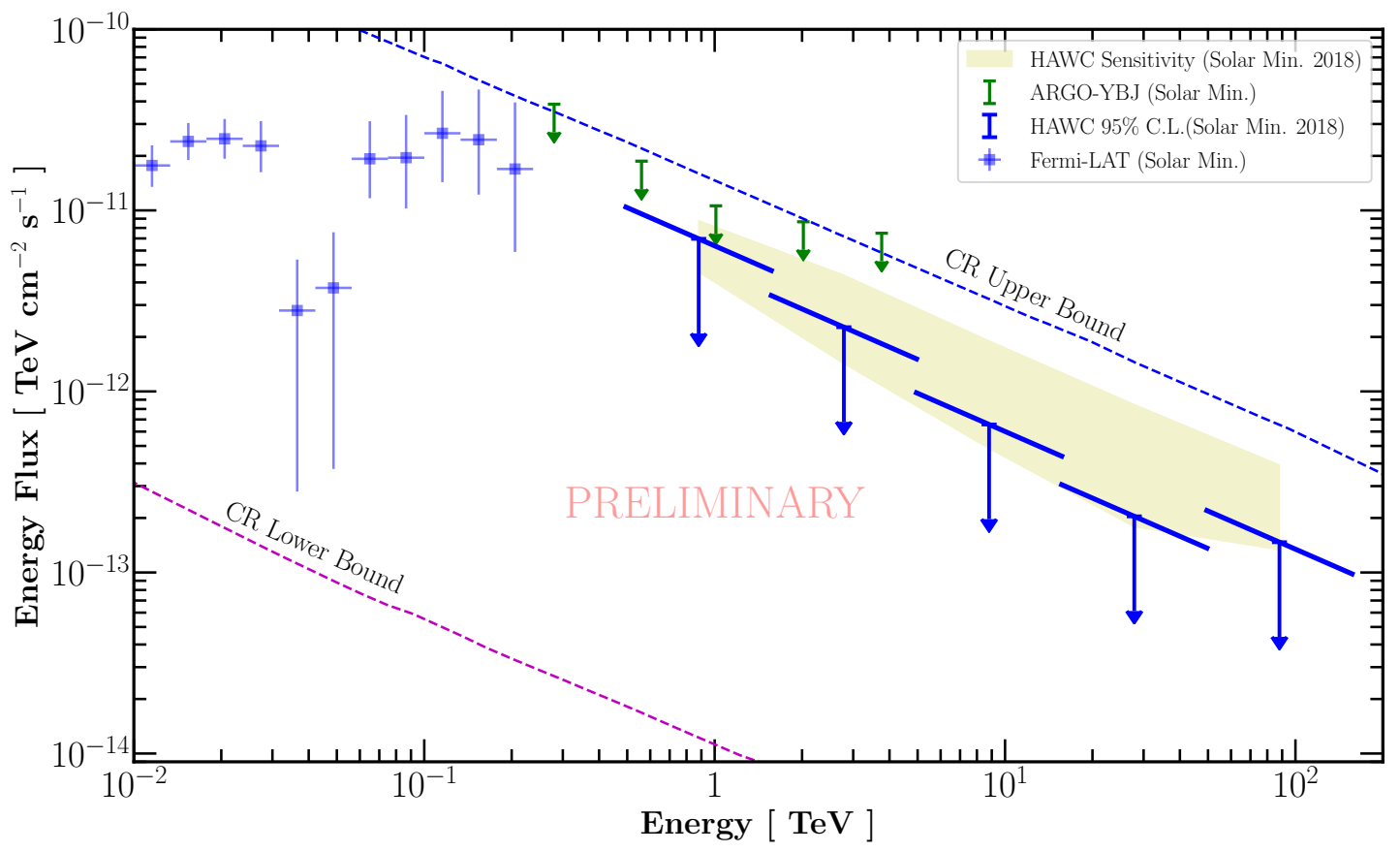

Figure 6: $95 \%$ C.L. upper limits and sensitivity for HAWC with one year of solar minimum data. The blue data points show the measured flux during the last solar minimum by the Fermi-LAT. Also shown are the upper limits obtained by the ARGO-YBJ [9] for the last solar minimum. The CR lower bound shows the minimum expected flux from the solar limb [10].

\subsection{Future Prospects}

We have presented a first search for TeV gamma rays from the Sun with HAWC as the solar cycle approaches a new solar minimum in 2018. The observed GeV flux from the Sun during the last solar minimum was much harder and brighter than during the solar maximum. The most useful solar minimum data from the Sun is going to be collected in 2019-2020. With more data in the current year, HAWC will be able to constrain the solar minimum spectrum of the Sun. Similarly, if the gamma-ray emission observed during the last solar minimum continues above $1 \mathrm{TeV}$, HAWC will be able to detect it. A full analysis of multiple years of the solar minimum data will be the subject of upcoming work.

\section{Acknowledgments}

We acknowledge the support from: the US National Science Foundation (NSF) the US Department of Energy Office of High-Energy Physics; the Laboratory Directed Research and Development (LDRD) program of Los Alamos National Laboratory; Consejo Nacional de Ciencia y Tecnología (CONACyT), México (grants 271051, 232656, 260378, 179588, 239762, 254964, 271737, 258865, 243290, 132197, 281653)(Cátedras 873, 1563, 341), Laboratorio Nacional HAWC de rayos gamma; L’OREAL Fellowship for Women in Science 2014; Red HAWC, México; DGAPA-UNAM (grants AG100317, IN111315, IN1117163, IA102715, IN109916, IA102019, IN112218); VIEP-BUAP; PIFI 2012, 2013, PROFOCIE 2014, 2015; the University of Wisconsin Alumni Research Foundation; the Institute of Geophysics, Planetary Physics, 
and Signatures at Los Alamos National Laboratory; Polish Science Centre grant DEC-2014/13/B/ST9/945, DEC-2017/27/B/ST9/02272; Coordinación de la Investigación Científica de la Universidad Michoacana; Royal Society - Newton Advanced Fellowship 180385. Thanks to Scott Delay, Luciano Díaz and Eduardo Murrieta for technical support.

Thanks to J.F. Beacom, A. Peter, T. Linden, K.C.Y. Ng, B. Zhou and R. Leane for helpful discussions leading up to this work.

\section{References}

[1] A. Abdo et al., "Fermi Large Area Telescope Observations of Two Gamma-Ray Emission Components from the Quiescent Sun," Astrophys. J., vol. 734, no. 2, p. 116, 2011.

[2] Q.-W. Tang, K. C. Y. Ng, T. Linden, B. Zhou, J. F. Beacom, and A. H. G. Peter, “An Unexpected Dip in the Solar Gamma-Ray Spectrum," ArXiv e-prints, Apr. 2018.

[3] K. C. Y. Ng, J. F. Beacom, A. H. G. Peter, and C. Rott, "First Observation of Time Variation in the Solar-Disk Gamma-Ray Flux with Fermi,” Phys. Rev., vol. D94, no. 2, p. 023004, 2016.

[4] T. Linden, B. Zhou, J. F. Beacom, A. H. G. Peter, K. C. Y. Ng, and Q.-W. Tang, "Evidence for a New Component of High-Energy Solar Gamma-Ray Production," ArXiv e-prints, Mar. 2018.

[5] "First Search for Steady TeV Gamma Rays from the Sun with HAWC," Phys. Rev., vol. D98, no. 12, p. 123011, 2018.

[6] A. U. Abeysekara et al., "Observation of the Crab Nebula with the HAWC Gamma-Ray Observatory," Astrophys. J., vol. 843, p. 39, July 2017.

[7] A. U. Abeysekara et al., "Observation of Small-scale Anisotropy in the Arrival Direction Distribution of TeV Cosmic Rays with HAWC," Astrophys. J., vol. 796, p. 108, Dec. 2014.

[8] M. G. Aartsen et al., "Detection of the Temporal Variation of the Sun's Cosmic Ray Shadow with the IceCube Detector,” Astrophys. J., vol. 872, no. 2, p. 133, 2019.

[9] B. Bartoli et al., "Search for gamma-ray emission from the Sun during solar minimum with the ARGOYBJ experiment," Astrophys. J., vol. 872, no. 2, p. 143, 2019.

[10] B. Zhou, K. C. Y. Ng, J. F. Beacom, and A. H. G. Peter, "TeV solar gamma rays from cosmic-ray interactions," Phys. Rev. D, vol. 96, p. 023015, July 2017. 